Citation: Lundgren S. et al. (2018)

"Electrocardiographic characteristics, antiarrhythmic utilization, and outcomes in patients with left ventricular assist devices".

The VAD Journal, 4. doi: https://doi.org/10.13023/vad.2018 .08

Editor-in-Chief: Maya Guglin, University of Kentucky

Received: July 6, 2018

Accepted: August 19, 2018

Published: August 19, 2018

(c) 2018 The Author(s). This is an open access article published under the terms of the Creative Commons Attribution-

NonCommercial 4.0 International License

(https://creativecommons.org/lice nses/by-nc/4.0/), which permits unrestricted non-commercial use, distribution, and reproduction in any medium, provided that the original author(s) and the publication source are credited.

Funding: Not applicable

Competing interests: Dr. Um discloses being a consultant for Abbott. Other authors have no relevant disclosures
Peer-Reviewed Original Research

\section{Electrocardiographic characteristics, antiarrhythmic utilization, and outcomes in patients with left ventricular assist devices}

\author{
Scott Lundgren*, Elizabeth Lyden, Douglas Stoller, Marshall Hyden, \\ Adam Burdorf, Ronald Zolty, John Um, and Brian Lowes
}

*Corresponding author: scott.lundgren@unmc.edu

University of Nebraska Medical Center, Omaha, NE

\begin{abstract}
Background

Left ventricular assist devices (LVAD) are an increasingly used therapy for patients with advanced heart failure. Arrhythmias are common complications following LVAD implantation requiring admission, initiation, and escalation of medical therapy. Despite their frequent use in the treatment of arrhythmias, little has been reported regarding electrocardiographic changes, antiarrhythmic utilization, and outcomes post-LVAD.
\end{abstract}

\section{Methods}

A total of 309 patients who received a LVAD underwent retrospective chart review pre- and post-LVAD. Kaplan-Meier curves were calculated and compared using the log-rank test. Cox regression model was used for univariate analysis and those with a $\mathrm{p}<0.15$ were included in multivariate analysis to evaluate for overall survival.

\section{Results}

There was a significant reduction in both the QRS interval $(p=0.0001)$ and QTc interval $(p=0.0074)$ following LVAD implantation. Ventricular tachycardia is common following LVAD implant at $31.1 \%$. Amiodarone use was frequent prior to LVAD (52.1\%) and on discharge (68.6\%). Amiodarone use ( $\mathrm{p}=0.019$, HR 1.7, 95\% Cl 1.1-2.6), age at implant $(p<0.001)$, creatinine $(p=0.004)$, albumin $(p=0.02)$, redcell distribution width $(p=0.047)$, and prior median sternotomy $(p<0.0001)$, were associated with increased mortality. On multivariate analysis, only albumin $(p=0.013)$ and prior median sternotomy $(p<0.001)$ remained statistically significant. There was no significant difference in post-operative duration of intubation, number of readmissions, or length of stay base on amiodarone utilization. 


\section{Conclusions}

Amiodarone is a commonly used antiarrhythmic in advanced heart failure and its use prior to LVAD implantation may increase the risk of long-term mortality. The amiodarone efficacy needs to be weighed against its long-term side effects and implant on clinical outcomes.

\section{Keywords: LVAD, antiarrhythmic, amiodarone, electrocardiography}

\section{Introduction}

While treatments for heart failure have improved over the last two decades, the number of patients with advanced heart failure who are or become refractory to standard medical therapy continues to increase. Heart transplantation alone is not possible for a large proportion of this increasing population of patients, due to an overall plateau in the number of organs available for transplantation worldwide as well as patient-specific factors making them poor candidates for heart transplantation. With the continued development and improvement in left ventricular assist device (LVAD) design, along with decreased morbidity and mortality post-implantation, the incidence of new device implantations not only as bridge to transplantation (BTT), but also as destination therapy (DT) has significantly increased over the last 15 years. ${ }^{1}$ Arrhythmias, especially ventricular arrhythmias, are a common post-LVAD complication, with an incidence ranging from $22-59 \%$ and are frequently better tolerated in LVAD patients due to their ability to maintain cardiac output when perturbations in heart rate and ventricular synchrony are present. ${ }^{2,3}$

Appropriate treatment of arrhythmias after LVAD implantation havs not been completely defined and have only been reported in a couple of small, retrospective studies. ${ }^{4,5}$ While antiarrhythmic medications are frequently used to treat both atrial and ventricular arrhythmias in LVAD patients, the use of these agents and the outcomes associated with their used have not been adequately described. The purpose of this study was to determine common rhythms/arrhythmias and electrocardiographic (ECG) changes related to LVAD implantation as well as antiarrhythmic utilization to treat these arrhythmias and how these impact outcomes following implantation.

\section{Methods}

\section{Study Population}

We conducted a retrospective chart review of 309 patients who underwent LVAD implantation as BTT or DT at The University of Nebraska Medical Center between July 2004 and October 2017. This involved a full review of the patient's electronic health records (EHR), including physician progress notes, nursing notes, telemetry records, and implantable cardioverter defibrillator (ICD) interrogations, evaluating for documented evidence of atrial or ventricular arrhythmias as well as patient's medication administration records to document prescribing of antiarrhythmic 
medications. Records were reviewed from the time of first preoperative evaluation by our cardiology or cardiothoracic surgery team and followed postoperatively until death, heart transplant, or completion of data collection on November $27^{\text {th }}, 2017$. The study was approved by the institutional review board at our institution.

\section{Clinical Variables}

We obtained demographic and clinical variables on patients undergoing LVAD implantation via review of the EHR. These variables included age, gender, left ventricular ejection fraction (LVEF), left ventricular internal diameter end diastole (LVIDd), ICD status, routine laboratory values (e.g. potassium, creatinine, albumin, etc.), antiarrhythmic therapy and other drug therapy pre- and post-LVAD implantation, and other pertinent medical history (e.g. presence of arrhythmias, pre-implantation comorbidities, etc.).

Ventricular tachycardia (VT) was defined as sustained or non-sustained VT (NSVT) with symptoms requiring antiarrhythmic therapy. Preoperative VT and atrial fibrillation (afib) were defined as documentation within clinical notes, telemetry records, or ECG of VT or afib prior to LVAD implantation. Postoperative VT or afib were defined as documented VT or afib within clinical notes, telemetry records, or ECG following LVAD implantation.

Pre- and post-LVAD ECG intervals (PR, QRS, QT, and QTC) and rhythms were obtained via review of each ECG documented in the patient's EHR. Both ventricular-paced and non-paced rhythms and intervals were documented for comparison between pre- and post-implantation periods.

\section{Statistical Analysis}

Descriptive statistics included counts and percentages for categorical data and means and standard deviations for continuous data. The independent sample ttest was used to compare continuous measures between groups. Overall survival (OAS) was defined as the time from implant to time of death from any cause. Patients who underwent heart transplantation were censored at the time of transplant and patients who underwent LVAD explantation or defunctionalization were censored at the time of this surgery. The OAS curve was calculated using the Kaplan-Meier method and compared using the log-rank test. Confidence interval for estimate of time-to-event distribution was calculated using Greenwood's formula. The Cox regression model for censored data was used to examine patient characteristics of OAS in a univariate analysis. Factors significant on univariate analysis $(p<0.15)$ were included in the multivariate analysis. For secondary outcomes, Fisher's exact test was used to compare intubation at 24 hours between groups and the Mann-Whitney test was used to compare the median LOS and hospital readmissions between groups. A p-value of $<0.05$ was considered statistically significant.

\section{Results}

Our study population included 309 patients, 240 (77.7\%) were males and the mean age was 56.7 (12.9) years. Destination therapy was the implant strategy for 
187 (60.5\%) patients and 163 (52.7\%) had ischemic cardiomyopathy. The mean left ventricular ejection fraction (LVEF) was $17.9 \%$ (8.9) and mean left ventricular internal dimension in diastole (LVIDd) was $64.7 \mathrm{~mm}$ (9.1). Patients on amiodarone prior to LVAD implantation tended to be older (59.2 vs 54 years, $p<0.0001$ ), more likely female $(25.8 \%$ vs $18.7 \%, p=0.04)$, have a history of chronic obstructive pulmonary disease ( $27 \%$ vs $16.7 \%)$, have a higher creatinine (1.37 vs 1.25 , $\mathrm{p}=0.01$ ), lower albumin (3.27 vs $3.31, \mathrm{p}=0.04$ ), and have higher prevalence of ventricular tachycardia $(62.9 \%$ vs $24.7 \%, p<0.0001)$ and atrial fibrillation $(71.7 \%$ vs $38 \%, \mathrm{p}<0.0001)$. Full pre-LVAD clinical characteristics are shown in Table 1.

Table 1. Pre-LVAD Implantation Patient Characteristics and Hemodynamics

\begin{tabular}{|c|c|c|c|c|}
\hline Characteristic & $\begin{array}{c}\text { Total } \\
\text { population, } \\
\mathrm{N}=309\end{array}$ & $\begin{array}{c}\text { Pre-LVAD } \\
\text { Amiodarone, } \\
\mathrm{N}=159\end{array}$ & $\begin{array}{c}\text { No Pre-LVAD } \\
\text { Amiodarone, } \mathrm{N}= \\
150\end{array}$ & p-value \\
\hline Age, y (SD) & $56.7(12.9)$ & $59.2(11.7)$ & $54.0(13.7)$ & $<0.0001$ \\
\hline Female Sex, N (\%) & $69(22.3)$ & $41(25.8)$ & $28(18.7)$ & 0.04 \\
\hline BTT Implant Strategy, N (\%) & $187(60.5)$ & $92(57.9)$ & $96(64)$ & 0.29 \\
\hline Pre-LVAD BMI, kg/m2 (SD) & $30.1(6.6)$ & $29.69(5.4)$ & $30.40(7.9)$ & 0.99 \\
\hline Ischemic Cardiomyopathy, N (\%) & $163(52.8)$ & $88(55.3)$ & $74(49.3)$ & 0.54 \\
\hline Baseline LVEF, \% (SD) & $17.9(8.9)$ & $19.5(10.3)$ & $17(8.4)$ & 0.034 \\
\hline Baseline LVIDd, mm (SD) & $64.7(9.1)$ & $64.1(9)$ & $65.1(9.4)$ & 0.25 \\
\hline \multicolumn{5}{|l|}{ INTERMACS Profile at Implantation } \\
\hline 1 & $63(20)$ & 31 & 32 & 0.69 \\
\hline 2 & $83(27)$ & 41 & 42 & 0.66 \\
\hline 3 & $99(32)$ & 50 & 49 & 0.82 \\
\hline 4 & $62(20)$ & 36 & 26 & 0.24 \\
\hline $5-7$ & $2(1)$ & 0 & 2 & 0.14 \\
\hline \multicolumn{5}{|l|}{ Comorbidities } \\
\hline Hypertension, N (\%) & $186(60.2)$ & $102(64.2)$ & $83(55.3)$ & 0.20 \\
\hline Diabetes Mellitus, N (\%) & $123(39.8)$ & $66(41.5)$ & $58(38.7)$ & 0.73 \\
\hline Obstructive Sleep Apnea, N (\%) & $92(29.8)$ & $52(32.7)$ & $40(26.7)$ & 0.27 \\
\hline COPD, N (\%) & $68(22)$ & $43(27)$ & $25(16.7)$ & 0.039 \\
\hline Chronic Kidney Disease, N (\%) & $99(32)$ & $57(35.8)$ & $42(28)$ & 0.18 \\
\hline Prior Median Sternotomy, N (\%) & $100(32)$ & $53(33.3)$ & 47 (31.3) & 0.72 \\
\hline Ventricular Tachycardia, N (\%) & $137(44.3)$ & $100(62.9)$ & $37(24.7)$ & $<0.0001$ \\
\hline Atrial Fibrillation, N (\%) & $171(55.3)$ & $114(71.7)$ & $57(38)$ & $<0.0001$ \\
\hline \multicolumn{5}{|l|}{ Lab Values } \\
\hline Hemoglobin, g/dL (SD) & $11.8(2.0)$ & $11.7(2)$ & $12(2)$ & 0.12 \\
\hline Creatinine, mg/dL (SD) & $1.3(0.4)$ & $1.37(0.45)$ & $1.25(0.41)$ & 0.01 \\
\hline Albumin, g/dL (SD) & $3.3(0.6)$ & $3.27(0.55)$ & $3.41(0.55)$ & 0.04 \\
\hline RDW, \% (SD) & $15.9(2.5)$ & $15.9(2.4)$ & $15.8(2.7)$ & 0.39 \\
\hline \multicolumn{5}{|l|}{ Pre-LVAD Hemodynamics } \\
\hline Right Atrial Pressure, mmHg (SD) & $12.5(6.3)$ & $12.8(6.4)$ & $12.3(6.2)$ & 0.56 \\
\hline Pulmonary Artery Saturation, \% (SD) & $53(10.3)$ & $53.2(10.7)$ & $52.7(10)$ & 0.65 \\
\hline Wedge Pressure, mmHg (SD) & $24(8.9)$ & $23.7(9.1)$ & $24.4(8.7)$ & 0.32 \\
\hline Fick Cardiac Index, L/min/m2 (SD) & $2(0.5)$ & $2.09(0.6)$ & $1.97(0.5)$ & 0.057 \\
\hline
\end{tabular}

$\mathrm{Y}=$ year; $\mathrm{DT}=$ destination therapy; $\mathrm{BMI}=$ body mass index; $\mathrm{SD}=$ standard deviation; $\mathrm{BSA}=$ body surface area; LVEF = left ventricular ejection fraction; LVIDd= left ventricular internal dimension in diastole; $\mathrm{RDW}$ = red blood cell distribution width; $\mathrm{TSH}=$ thyroid stimulating hormone; $\mathrm{ICD}=$ implantable cardioverter defibrillator; CRT-D = cardiac resynchronization therapy-defibrillator 
Of the 290 patients who survived to discharge, 143 (49.3\%) patients had CRT-D in place, with no new patients receiving or being upgraded to CRT-D following LVAD implantation. A total of $135(46.6 \%)$ patients had an ICD in place at the time of discharge compared to $83(28.6 \%)$ patients at the time of index admission. Nine patients were discharged from the hospital without an ICD in place. ECGs were evaluated both pre- and post-LVAD implantation. A total of 76 patients had a nonventricularly paced ECG within 1 month prior to LVAD implantation and a nonventricularly paced ECG 3 or more months following LVAD implantation for comparison of intervals pre- and post-LVAD (Table 2).

Table 2. Comparison of Non-Ventricular Paced ECG Intervals Pre- and PostLVAD Implantation

\begin{tabular}{|l|c|c|c|}
\hline ECG Interval & $\begin{array}{c}\text { Average Pre-LVAD } \\
\text { Duration, } \mathrm{ms}(\mathrm{SD})\end{array}$ & $\begin{array}{c}\text { Average Post-LVAD } \\
\text { Duration, } \mathrm{ms}(\mathrm{SD})\end{array}$ & P value $(95 \% \mathrm{Cl})$ \\
\hline PR & 174.3 & 169.5 & $0.23(-3.9-16.0)$ \\
\hline QRS & 115.2 & 102.9 & $0.0001(6.6-17.4)$ \\
\hline QT & 393.9 & 406.4 & $0.14(-29.2-4.2)$ \\
\hline QTc (Bazett) & 485.6 & 466.8 & $0.0074(5.2-32.5)$ \\
\hline
\end{tabular}

Of the 309 patients in our study, 137 (44.3\%) patients had documented NSVT or sustained VT prior to undergoing LVAD implantation. Thirty-nine (12.6\%) patients were admitted to the hospital for their index admission because of VT or had VT during their admission, but prior to undergoing LVAD implantation. The most common pre- and post-LVAD rhythm was a ventricular-paced rhythm (Vpaced) with $118(38.2 \%)$ patients having this as their primary rhythm prior to implantation and $111(35.9 \%)$ having this as their primary rhythm post-implantation (Table 3 ).

Table 3. Most Common Heart Rhythms Pre- and Post-LVAD Implantation

\begin{tabular}{|l|c|c|}
\hline Rhythm & Pre-LVAD, N (\%) & Post-LVAD, N (\%) \\
\hline Ventricular Paced & $118(38.1)$ & $111(36.0)$ \\
\hline Normal Sinus Rhythm & $106(34.3)$ & $103(33.3)$ \\
\hline Sinus Tachycardia & $48(15.5)$ & $33(10.7)$ \\
\hline Afib/Aflutter & $17(5.5)$ & $21(6.8)$ \\
\hline Unknown Rhythm & $8(2.6)$ & $37(12.0)$ \\
\hline Atrial Paced & $7(2.3)$ & $4(1.3)$ \\
\hline Sinus Bradycardia & $5(1.6)$ & $0(0.0)$ \\
\hline
\end{tabular}

Atrial fibrillation or atrial flutter were present in 171 (55.3\%) patients prior to LVAD implantation and $120(38.8 \%)$ patients post-LVAD, but these were the primary rhythm in only $17(5.5 \%)$ patients pre-LVAD and 21 (6.8\%) patients post-LVAD. A total of $98(31.7 \%)$ patients had a ventricular arrhythmia following LVAD implantation. Ninety-three (94.9\%) had VT only, $2(2 \%)$ patients had VF only, and $3(3 \%)$ patients had both VT and VF following implantation. Of the 98 patients who had a ventricular arrhythmia post-LVAD, $48(49 \%)$ patients had a history of ventricular arrhythmia pre-LVAD, while the other $50(51 \%)$ patients had a 
ventricular arrhythmia only after LVAD. While 12 (3.9\%) patients underwent VT ablation prior to LVAD implantation, only $1(0.3 \%)$ patient had a VT ablation in the post-implantation period. Frequent post-implantation VT and limited VT ablation led to frequent prescribing of antiarrhythmics post-LVAD, with $225(72.8 \%)$ patients being prescribed at least one antiarrhythmic in the post-implantation period. Only $24(7.8 \%)$ patients were on multiple antiarrhythmic concurrently following LVAD implantation. A total of $161(52.1 \%)$ patients were on amiodarone within 2 years prior to LVAD implantation and 158 (51.4\%) patients who survived to discharge were continued on amiodarone at the time of their dismissal. Drug utilization frequencies pre- and post-implantation are listed in Table 4.

Table 4. Drug Utilization Pre- and Post-LVAD Implantation

\begin{tabular}{|l|c|}
\hline \multicolumn{1}{|c|}{ Medication } & Utilization \\
\hline On beta blocker at time of LVAD admission, N (\%) & $225(72.8)$ \\
\hline On amiodarone within 2 years prior to LVAD, N (\%) & $159(51.4)$ \\
\hline Discharged on beta blocker post-LVAD, N (\%) & $76(27.7)$ \\
\hline Discharged on digoxin post-LVAD, N (\%) & $110(40.1)$ \\
\hline Discharged on amiodarone post-LVAD, N (\%) & $158(57.7)$ \\
\hline On beta blocker 3 months post-LVAD, N (\%) & $89(34.9)$ \\
\hline Antiarrhythmic therapy post-LVAD, N (\%) & $225(72.8)$ \\
\hline Amiodarone use at any point post-LVAD, N (\%) & $212(68.6)$ \\
\hline Post-LVAD Mexiletine, N (\%) & $18(5.8)$ \\
\hline Post-LVAD Sotalol, N (\%) & $12(3.9)$ \\
\hline Post-LVAD Dofetilide, N (\%) & $5(1.6)$ \\
\hline Post-LVAD Quinidine, N (\%) & $2(0.6)$ \\
\hline
\end{tabular}

On univariate analysis, amiodarone was associated with increased mortality, with a hazard ratio of $1.699(\mathrm{P}=0.0193, \mathrm{Cl} 1.090-2.648)$. The Kaplan-Meier survival curve is displayed in Figure 1. Other variables associated with increased mortality include age at implant $(p=0.0006)$, creatinine $(p=0.0042)$, albumin $(p=0.022)$, RDW $(p=0.0469)$, and prior median sternotomy $(p<0.0001)$ (Table 5$)$.

\section{Table 5. Univariate Analysis of Pre-LVAD Variables and Their Impact On Survival}

\begin{tabular}{|l|c|c|c|}
\hline \multicolumn{1}{|c|}{ Variable } & $\begin{array}{c}\text { Hazard } \\
\text { Ratio }\end{array}$ & P-Value & $\begin{array}{c}\text { 95\% Confidence } \\
\text { Interval }\end{array}$ \\
\hline Pre-LVAD amiodarone & 1.699 & 0.019 & $1.09-2.65$ \\
\hline Age at Implant & 1.04 & 0.0006 & $1.02-1.06$ \\
\hline BMI at Implant & 0.99 & 0.68 & $0.96-1.03$ \\
\hline Pre-LVAD Creatinine & 1.97 & 0.004 & $1.24-3.14$ \\
\hline Pre-LVAD Albumin & 0.63 & 0.022 & $0.43-0.94$ \\
\hline Pre-LVAD RDW & 1.08 & 0.047 & $1.001-1.17$ \\
\hline Pre-LVAD COPD & 1.13 & 0.63 & $0.69-1.84$ \\
\hline Prior Pre-LVAD sternotomy & 2.30 & $<0.0001$ & $1.57-3.73$ \\
\hline
\end{tabular}




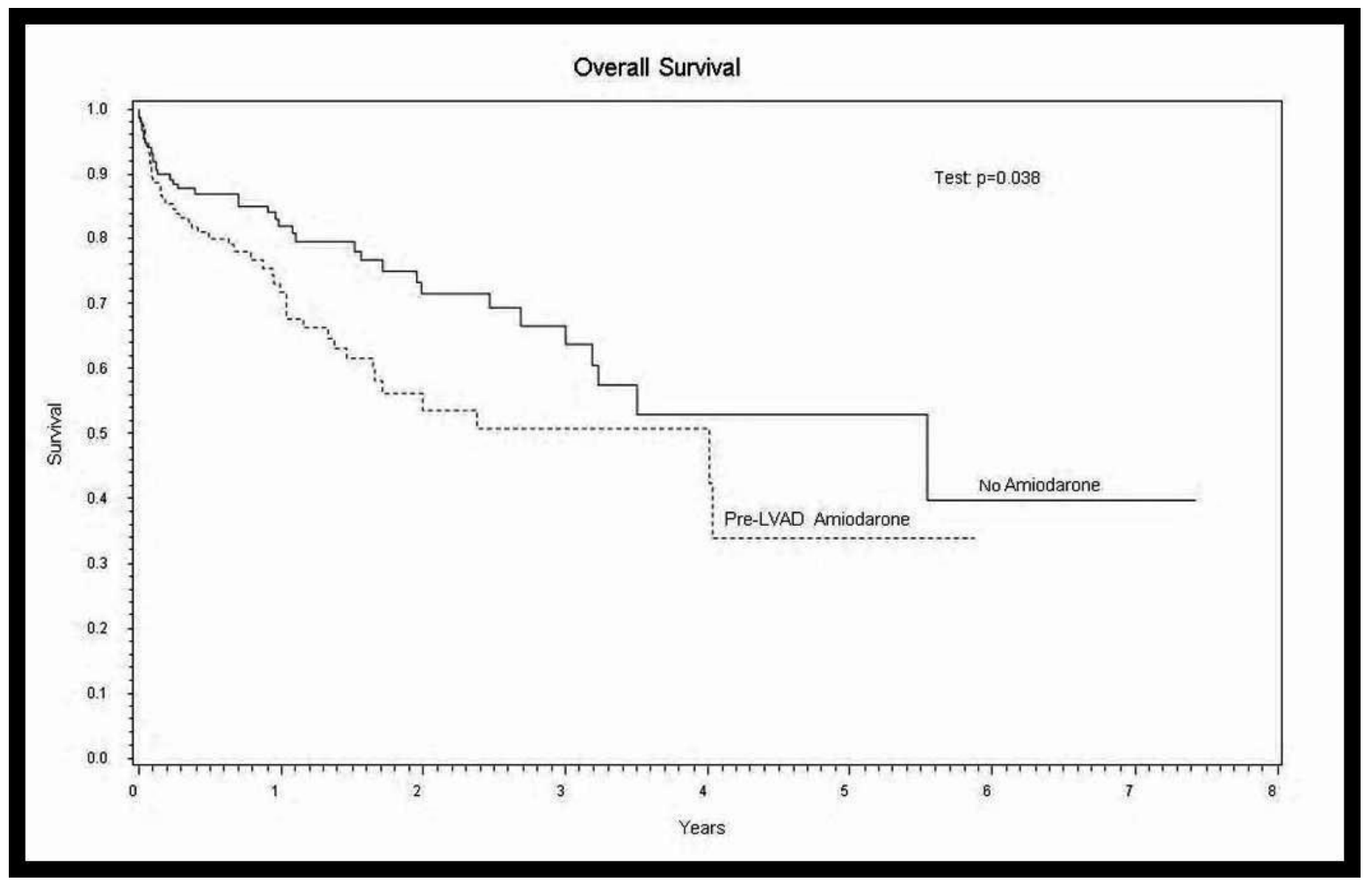

Figure 1. Kaplan-Meier survival curve showing worse post-implantation survival in patients who were on amiodarone prior to LVAD compared to those who were not on Amiodarone

On multivariate analysis, only albumin $(p=0.03)$ and prior median sternotomy $(p<0.001)$ remained independent predictors of survival (Table 6$)$. The use of amiodarone prior to LVAD implantation did not have an impact on duration of intubation following LVAD placement $(p=0.43)$, length of stay following LVAD implantation $(p=0.10)$, or number of readmissions $(p=0.57)$.

Table 6. Multivariate Analysis of Pre-LVAD Variables and Their Impact on Survival

\begin{tabular}{|l|c|c|c|}
\hline \multicolumn{1}{|c|}{ Variable } & $\begin{array}{c}\text { Hazard } \\
\text { Ratio }\end{array}$ & P-Value & $\begin{array}{c}\text { 95\% Confidence } \\
\text { Interval }\end{array}$ \\
\hline Pre-LVAD amiodarone use & 1.5 & 0.09 & $0.94-2.38$ \\
\hline Age at Implant & 1.0 & 0.16 & $0.99-1.04$ \\
\hline Pre-LVAD Creatinine & 1.5 & 0.11 & $0.91-2.57$ \\
\hline Pre-LVAD Albumin & 0.6 & 0.03 & $0.42-0.96$ \\
\hline Pre-LVAD RDW & 1.0 & 0.35 & $0.96-1.1$ \\
\hline Prior Pre-LVAD sternotomy & 2.3 & $<0.001$ & $1.41-3.62$ \\
\hline
\end{tabular}




\section{Discussion}

This single center, retrospective study showed that patients with a history of amiodarone use within two years of LVAD implantation were at increased risk of post-operative mortality, as were older patients, those with prior median sternotomies, low albumin, and high creatinine and RDW. On multivariate analysis, only albumin and prior median sternotomy remained independent predictors of mortality. This study also showed that mechanical unloading of the heart leads to a significant reduction in the QRS and QTc intervals. We found that ventricular tachycardia is common in end-stage heart failure, both pre- and post-LVAD implantation and amiodarone is the most commonly used antiarrhythmic used to treat arrhythmias before and after LVAD implantation.

ECGs were evaluated both pre- and post-LVAD implantation for rhythm and interval durations. A total of 76 patients had non-ventricularly paced ECGs both pre- and post-LVAD implantation available for comparison. We found a statistically significant decrease in QRS duration $(p=0.0001)$ as well as a significant decrease in the QTc interval ( $p=0.0074)$ from pre- to post-implantation. Harding and colleagues previously looked at changes in ECG intervals pre- and post-LVAD and found a significant decrease in the QRS immediately post-implant (within 6 hours), but this was not sustained more than a week post-LVAD. ${ }^{6}$ It has been hypothesized that since changes in conduction velocity after cardiac distension have not been identified, possible decreases in cardiac dimensions post-LVAD lead to an overall decrease in conduction distance and thus a decrease in QRS duration. This same group has also noted a significant increase in QT and QTc intervals in the immediate post-implantation period followed by a significant decrease in these intervals more than 1-week post-implantation. ${ }^{7,8}$ While we did not evaluate ECGs in the immediate post-implantation period, we did identify a significant decrease in the QTc interval when evaluating a more delayed effect of mechanical unloading on ECG characteristics. Action potential prolongation has previously been shown to be a common abnormality in failing hearts and it has been hypothesized that mechanical unloading of failing hearts reverses this characteristic resulting in decreased action potential durations and thus, shorter QTc intervals. ${ }^{9}$

The primary rhythm identified on ECG and telemetry review in our study was $\mathrm{V}$ paced, both in the pre-LVAD (38.1\%) and post-LVAD (36\%) time periods. Martinez and colleagues have previously shown a similar prevalence of V-paced rhythm in their pre-implantation population at $40 \%$, but the percentage of patients primarily V-paced following implantation increased to $79 \%$ in their study. Normal sinus rhythm and sinus tachycardia were the next two most common rhythms both preand post-implantation. There was a low number of patients without ECGs obtained in the pre-LVAD period (2.8\%) and significantly higher in the post-implantation period (12\%). Martinez and colleagues have emphasized the importance of obtaining an ECG in every patient in the post-implantation period to establish a new baseline. ${ }^{10}$ While most of the rhythm monitoring in the post-implantation period is done via ICD interrogation, ECGs are still clinically useful for monitoring 
of the QT/QTc duration, especially in patients receiving antiarrhythmic therapy and potentially identifying patients at risk for ventricular arrhythmias.

Our study showed that $31.3 \%$ of patients had VT following LVAD implantation, which is in line with previous studies that have found that VT occurred anywhere from $22-59 \%$ following LVAD implantation. ${ }^{11,12}$ We also found that $44.3 \%$ of patients had at least one episode of VT prior to undergoing LVAD implantation with $12.6 \%$ of patients having an episode of VT leading to index admission or during the in-hospital evaluation prior to implantation. Afib was a problem for $55.3 \%$ of patients prior to LVAD, but only $38.8 \%$ of patients continued to have issues with afib following implantation. A previous study by Enriquez and colleagues showed a slightly higher incidence of afib post-LVAD at $51.9 \% .{ }^{13}$ They also found that persistent afib was an independent predictor of death and hospitalization for heart failure. While previous studies have shown that ventricular arrhythmias tend to occur within the first week to month following LVAD-implantation, ${ }^{4,14}$ timing of ventricular arrhythmias was not evaluated in our study.

We found that $49 \%$ of patients had CRT in place prior to undergoing LVAD implantation and this did not change following implantation. While only $26.9 \%$ of patients had a single-chamber ICD in place prior to LVAD implantation, 52 new devices were implanted in the post-implantation period. In our study, $75.7 \%$ of patients had an ICD in place (whether biventricular or single chamber) at the time of LVAD implantation. Previous studies have had significant variability in the number of patients with ICDs in place prior to LVAD implantation, ranging anywhere from $18.8 \%$ and $31.1 \%$, up to $75.5 \% .{ }^{15-17}$ Our study is more in line with data presented by Enriquez and colleagues, with $75.7 \%$ having an ICD in place at the time of LVAD, likely indicating that our patients tend to have more chronic heart failure with attempts at optimizing their medical and device therapy prior to proceeding with LVAD and transplant evaluation. ${ }^{17}$

There was a total of nine patients who were discharged without an ICD in place. The reasons for not placing an ICD in these patients varied and included: 1.) prior ICD explantation due to infection, 2.) two with improvement in left ventricular function or decrease in ectopy prior to discharge, 3.) electrophysiology study showing no inducible VT, 4.) receipt of heart transplantation prior to initial discharge, 5.) concern for hypotension with anesthesia and risk for neurologic event, and 6.) three patients with unclear documentation as to why no ICD was placed. To our knowledge, there are no studies investigating the use of intracardiac EP studies in the post-LVAD population to assess low-risk patients for inducible VT to help direct ICD placement. A recent meta-analysis of six observational studies totaling 937 patients showed ICD use in LVAD patients was associated with a significant reduction in mortality, but in a subgroup analysis of 361 patients with continuous flow LVADs, there was not a statistically significant decrease in mortality following LVAD implantation with an ICD in place. ${ }^{18}$

Antiarrhythmic medications are frequently used to treat both atrial and ventricular arrhythmias in advanced heart failure patients both before and after LVAD implantation. We found that $72.8 \%$ of patients were on at least one antiarrhythmic medication in the post-implantation period. Amiodarone was the most commonly used antiarrhythmic, with $94 \%$ of the patients requiring antiarrhythmic therapy 
being on amiodarone. Amiodarone use was equally frequent in the pre-LVAD period $(52 \%)$ and in the post-LVAD period, with $51 \%$ of patients being discharged from the hospital on amiodarone following LVAD implantation. Previous studies have also shown that amiodarone is the most commonly used antiarrhythmic in the post-LVAD setting, with $66.7-75 \%$ of antiarrhythmic utilization for VT being done with amiodarone..$^{11,14}$ The utilization of beta-blockers at discharge and 3 months following LVAD implantation is low in our institution (Table 4), historically due to concern for increased risk of right ventricular failure. Wever-Pinzon and colleagues recently showed that patients on a beta-blocker at 12 months following LVAD implantation were more likely to experience cardiac recovery and small, retrospective studies have shown it may improve post-implantation survival and does not appear to increase the number of heart failure readmissions. ${ }^{19-21}$

The side effects of long-term amiodarone use have been documented since the early 1980 s and primarily involve the thyroid gland, liver, lungs, skin, and cornea. ${ }^{22}$ Given amiodarone's frequent usage in patients undergoing heart transplant evaluation because of increased incidence of ventricular arrhythmias, a few studies over the last 20 years have looked at postoperative outcomes following heart transplantation in patients who received amiodarone in the pre- and postoperative period. Chin et al published a retrospective review in 1999 of 106 consecutive heart transplants and found that patients who had received amiodarone for more than 4 weeks pre-transplant had higher mortality at 1 year. ${ }^{23}$ Blomberg and colleagues found that patients who had received amiodarone within 3 months of heart transplant had decreased survival post-transplant, required longer periods of postoperative ventilatory support, and were more likely to experience bleeding complications. ${ }^{24}$ More recently, Cooper and colleagues published a retrospective cohort analysis of 14,955 patients from the International Society for Heart and Lung Transplantation registry that again found that patients treated with amiodarone prior to heart transplant had higher 1-year mortality but did not find increased risks of early graft failure, retransplantation, or rehospitalizations. ${ }^{25}$ To our knowledge, there have not been prior reports looking at amiodarone utilization prior to LVAD implantation and its impact on post-operative outcomes.

Despite 137 patients having VT prior to LVAD implantation and 96 patients having VT post-implantation, only 12 patients underwent VT ablation pre-LVAD and 1 patient post-LVAD at our institution. Per-LVAD and post-LVAD VT ablation have previously been well described in several studies. Garan and colleagues looked at 224 patients who underwent LVAD placement and identified 7 patients who underwent EP study and VT ablation following LVAD implantation. ${ }^{26}$ Multiple VTs were identified and ablated, with 5 of the 7 patients having a significant reduction in their VT following ablation. ${ }^{26} \mathrm{~A}$ larger study evaluated 611 patients who underwent LVAD implantation over almost a 20-year period and identified 21 patients who underwent post-LVAD EP study. Of the 20 patients who had inducible VT, 18 had acute ablative success with 7 patients having recurrent VT and underwent a repeat procedure. ${ }^{27}$ Amiodarone use decreased in both studies post-ablation with each study noting very few procedural complications and low post-procedural morbidity and mortality. Intraoperative ablation has been evaluated in a study of 5 patients who underwent peri-LVAD open chest hybrid epicardial mapping and VT ablation with 3 of the 5 patients having acute 
procedural success and no arrhythmia related deaths in any of the patients over a mean follow-up period of $363 \pm 368$ days. ${ }^{28}$

On a univariate analysis, we found that patients on amiodarone within two years prior to LVAD implantation had an increased risk of mortality, with a HR of 1.7 and $p=0.019$. We also found that pre-implantation albumin, creatinine, and RDW as well as patient's age and history of prior median sternotomy predicted an increased risk of mortality on univariate analysis. Our findings confirm prior studies showing increased risk of mortality with lower albumin, higher RDW, worse renal function, and increasing age at the time of implantation. ${ }^{29-34}$ Our findings of increased risk of mortality in patients with a history of a previous sternotomy prior to LVAD implantation conflict with a recent study published by Papathanasiou and colleagues. In their study of 112 patients who underwent implantation with HeartWare (HeartWare International Inc., Framingham, MA), they found no difference in survival rate at 6 months $(p=0.37)$ in patients who had a history of prior median sternotomy at the time of LVAD implantation compared to those without prior sternotomy. ${ }^{35}$ In our study, history of prior median sternotomy was the strongest predictor of increased mortality $(p<0.0001)$ and on multivariate analysis remained an independent predictor of mortality $(p=0.0007)$. Pre-LVAD albumin $(p=0.0302)$ was the only other independent predictor of increased mortality on multivariate analysis, similar to findings previously described by Kato and colleagues.

There are several limitations to our study. This is a single-center experience involving both DT and BTT patients. Our analysis is retrospective in nature with a limited patient population. In some cases, pre- and post-LVAD ECGs were not available and prevented full evaluation of the entire patient population. Transition to a new electronic health record during the study period may have created difficulties in transfer of information between the systems as well as documentation early on.

In summary, the present study shows a significant decrease in QRS and QTC intervals post-LVAD implantation. Amiodarone was frequently used in both the preand post-LVAD setting to treat both atrial and ventricular arrhythmias. Amiodarone use may increase post-LVAD mortality. Despite the incidence of VT both before and after LVAD implantation, ablation was performed relatively infrequently in our patient population. With numerous studies showing increased mortality following heart transplant in patients who received pre-transplant amiodarone and our study showing pre-LVAD amiodarone potentially being associated with worse survival on univariate analysis, further studies need to be done investigating amiodarone reduction strategies and if ablation for recurrent VT is a superior strategy in patients undergoing transplant evaluation or with LVADs as bridge to transplant. 


\section{References}

1. Lampropulos JF, Kim N, Wang Y, Desai MM, Barreto-Filho JAS, Dodson JA, Dries DL, Mangi AA, Krumholz HM. Trends in left ventricular assist device use and outcomes among medicare beneficiaries, 2004-2011. Open Heart 2014 August $01 ; 1(1)$.

2. Nakahara S, Chien C, Gelow J, Dalouk K, Henrikson CA, Mudd J, Stecker EC. Ventricular arrhythmias after left ventricular assist device. Circ Arrhythm Electrophysiol 2013 Lippincott Williams \& Wilkins;6(3):648-54.

3. Oz MC, Rose EA, Slater J, Kuiper JJ, Catanese KA, Levin HR. Malignant ventricular arrhythmias are well tolerated in patients receiving long-term left ventricular assist devices. J Am Coll Cardiol 1994 Dec;24(7):1688-91.

4. Refaat M, Chemaly E, Lebeche D, Gwathmey JK, Hajjar RJ. Ventricular arrhythmias after left ventricular assist device implantation. PACE - Pacing and Clinical Electrophysiology 2008;31(10):1246-52.

5. Andersen M, Videbæk R, Boesgaard S, Sander K, Hansen PB, Gustafsson F. Incidence of ventricular arrhythmias in patients on long-term support with a continuous-flow assist device (HeartMate II). The Journal of Heart and Lung Transplantation 2009 7;28(7):733-5.

6. Harding JD, Piacentino V, Gaughan JP, Houser SR, Margulies KB. Electrophysiological alterations after mechanical circulatory support in patients with advanced cardiac failure. Circulation 2001 Lippincott Williams \& Wilkins;104(11):1241-7.

7. Reiter MJ, Landers M, Zetelaki Z, Kirchhof CJ, Allessie MA. Electrophysiological effects of acute dilatation in the isolated rabbit heart: Cycle length-dependent effects on ventricular refractoriness and conduction velocity. Circulation 1997 Dec 2;96(11):4050-6.

8. Zhu WX, Johnson SB, Brandt R, Burnett J, Packer DL. Impact of volume loading and load reduction on ventricular refractoriness and conduction properties in canine congestive heart failure. J Am Coll Cardiol 1997 Sep;30(3):825-33.

9. Wickenden AD, Kaprielian R, Kassiri Z, Tsoporis JN, Tsushima R, Fishman GI, Backx $\mathrm{PH}$. The role of action potential prolongation and altered intracellular calcium handling in the pathogenesis of heart failure. Cardiovasc Res 1998 Feb;37(2):312-23.

10. Martinez SC, Fansler D, Lau J, Novak EL, Joseph SM, Kleiger RE. Characteristics of the electrocardiogram in patients with continuous-flow left ventricular assist devices. Annals of Noninvasive Electrocardiology 2015;20(1):628. 
11. Bedi M, Kormos R, Winowich S, McNamara DM, Mathier MA, Murali S. Ventricular arrhythmias during left ventricular assist device support. Am J Cardiol 2007 Apr 15;99(8):1151-3.

12. Harding JD, Piacentino III V, Rothman S, Chambers S, Jessup M, Margulies KB. Prolonged repolarization after ventricular assist device support is associated with arrhythmias in humans with congestive heart failure. J Card Fail 2005 4;11(3):227-32.

13. Enriquez AD, Calenda B, Gandhi PU, Nair AP, Anyanwu AC, Pinney SP. Clinical impact of atrial fibrillation in patients with the HeartMate II left ventricular assist device. J Am Coll Cardiol 2014 Nov 4;64(18):1883-90.

14. Ziv O, Dizon J, Thosani A, Naka Y, Magnano AR, Garan H. Effects of left ventricular assist device therapy on ventricular arrhythmias. J Am Coll Cardiol 2005;45(9):1428-34.

15. Cantillon DJ, Tarakji KG, Kumbhani DJ, Smedira NG, Starling RC, Wilkoff BL. Improved survival among ventricular assist device recipients with a concomitant implantable cardioverter-defibrillator. Heart Rhythm 2010 Apr;7(4):466-71.

16. Refaat MM, Tanaka T, Kormos RL, McNamara D, Teuteberg J, Winowich S, London B, Simon MA. Survival benefit of implantable cardioverter-defibrillators in left ventricular assist device-supported heart failure patients. J Card Fail 2012 Feb;18(2):140-5.

17. Enriquez AD, Calenda B, Miller MA, Anyanwu AC, Pinney SP. The role of implantable cardioverter-defibrillators in patients with continuous flow left ventricular assist devices. Circulation: Arrhythmia and Electrophysiology 2013;6(4):668-74.

18. Vakil K, Kazmirczak F, Sathnur N, Adabag S, Cantillon DJ, Kiehl EL, Koene R, Cogswell R, Anand I, Roukoz H. Implantable cardioverter-defibrillator use in patients with left ventricular Assist Devices. JACC: Heart Failure 2016 American College of Cardiology;4(10):772-9.

19. Wever-Pinzon O, Drakos SG, McKellar SH, Horne BD, Caine WT, Kfoury AG, Li DY, Fang JC, Stehlik J, Selzman $\mathrm{CH}$. Cardiac recovery during long-term Left Ventricular assist device support. Journal of the American College of Cardiology 2016 American College of Cardiology;68(14):1540-53.

20. Vaidya G, Salgado BC, Pillarella J, Dunbar-Matos C, Vijayakrishnan R, Lenneman A, Slaughter MS, Birks E, Abramov D. Beta blocker use in patients with LVADs is associated with lower proBNP and is not associated with heart failure hospitalizations or an increase in adverse events. The Journal of Heart and Lung Transplantation 2017 04/01; 2018/08;36(4):S169-70.

21. Yin M, Ko Y, Pekarek A, Wittersheim K, Cole RT, Gupta D, Nguyen D, Laskar $\mathrm{R}$, Smith A, Vega JD, et al. 054 - B-blockers improves overall survival during 
support with continuous-flow left ventricular assist device. Journal of Cardiac Failure 2016 August 2016;22(8, Supplement):S21.

22. Harris L, McKenna WJ, Rowland E, Holt DW, Storey GC, Krikler DM. Side effects of long-term amiodarone therapy. Circulation 1983 Jan;67(1):45-51.

23. Chin C, Feindel C, Cheng D. Duration of preoperative amiodarone treatment may be associated with postoperative hospital mortality in patients undergoing heart transplantation. J Cardiothorac Vasc Anesth 1999 10;13(5):562-6.

24. Blomberg PJ, Feingold AD, Denofrio D, Rand W, Konstam MA, Estes III NAM, Link MS. Comparison of survival and other complications after heart transplantation in patients taking amiodarone before surgery versus those not taking amiodarone. Am J Cardiol 2004 2/1;93(3):379-81.

25. Cooper LB, Mentz RJ, Edwards LB, Wilk AR, Rogers JG, Patel CB, Milano CA, Hernandez AF, Stehlik J, Lund LH. Amiodarone use in patients listed for heart transplant is associated with increased 1-year post-transplant mortality. J Heart Lung Transplant 2017 Feb;36(2):202-10.

26. Garan AR, Levin AP, Topkara V, Thomas SS, Yuzefpolskaya M, Colombo PC, Takeda K, Takayama H, Naka Y, Whang W, et al. Early post-operative ventricular arrhythmias in patients with continuous-flow left ventricular assist devices. J Heart Lung Transplant 2015 Dec;34(12):1611-6.

27. Cantillon DJ, Bianco C, Wazni OM, Kanj M, Smedira NG, Wilkoff BL, Starling $\mathrm{RC}$, Saliba WI. Electrophysiologic characteristics and catheter ablation of ventricular tachyarrhythmias among patients with heart failure on ventricular assist device support. Heart Rhythm 2012 Jun;9(6):859-64.

28. Patel M, Rojas F, Shabari Fr, Simpson L, Cohn W, Frazier Oh, Mallidi H, Cheng J, Mathuria N. Safety and feasibility of open chest epicardial mapping and ablation of ventricular tachycardia during the period of left ventricular assist device implantation. J Cardiovasc Electrophysiol 2016;27(1):95-101.

29. Kato TS, Kitada S, Yang J, Wu C, Takayama H, Naka Y, Farr M, Mancini DM, Schulze PC. Relation of preoperative serum albumin levels to survival in patients undergoing left ventricular assist device implantation. Am J Cardiol $2013 \mathrm{Nov}$ $1 ; 112(9): 1484-8$.

30. Miller PE, Houston BA, Schneider AL, Bush AL, Whitman GJ, Stevens GR, Tedford RJ, Russell SD. Associations of preimplant red blood cell distribution width with clinical outcomes among individuals with left ventricular assist devices. Asaio J 2016 Nov/Dec;62(6):677-83.

31. Sandner SE, Zimpfer D, Zrunek P, Rajek A, Schima H, Dunkler D, Zuckermann AO, Wieselthaler GM. Age and outcome after continuous-flow left ventricular assist device implantation as bridge to transplantation. J Heart Lung Transplant 2009 Apr;28(4):367-72. 
32. Sandner SE, Zimpfer D, Zrunek P, Rajek A, Schima H, Dunkler D, Grimm M, Wolner E, Wieselthaler GM. Renal function and outcome after continuous flow left ventricular assist device implantation. Ann Thorac Surg 2009 Apr;87(4):1072-8.

33. Yoshioka D, Sakaguchi T, Saito S, Miyagawa S, Nishi H, Yoshikawa Y, Fukushima S, Saito T, Daimon T, Ueno T, et al. Predictor of early mortality for severe heart failure patients with left ventricular assist device implantation: Significance of INTERMACS level and renal function. Circ J 2012;76(7):1631-8.

34. Coffin ST, Waguespack DR, Haglund NA, Maltais S, Dwyer JP, Keebler ME. Kidney dysfunction and left ventricular assist device support: A comprehensive perioperative review. Cardiorenal Med 2015 Feb;5(1):48-60.

35. Papathanasiou M, Tsourelis L, Pizanis N, Koch A, Kamler M, Rassaf T, Luedike $P$. Resternotomy does not adversely affect outcome after left ventricular assist device implantation. Eur J Med Res 2017 Nov 15;22(1):46,017-0289-2. 